\title{
Distintos enfoques adoptados por los médicos para tratar la nefrolitiasis en pediatría: estudio basado en una encuesta
}

\author{
Different approaches among physicians to treat pediatric stone \\ disease: a survey-based study
}

\author{
Prof. Dr. Bülent Önal ${ }^{a}$, Dra. Elif A. Kırlı, Prof. Asoc. Dra. Nur Canpolat ${ }^{b}$ \\ Prof. Asoc. Dr. Mehmet Taşdemirc, Dr. Ahmet Gürbüzza, Dr. Oktay Özman ${ }^{a}$, \\ Prof. Dra. Lale Sever ${ }^{b}$, Prof. Dra. Ilmay Bilge $e^{d}$ Prof. Dr. Salim Çalışkan ${ }^{b}$
}

a. Departamento de Urología, Facultad de Medicina de Cerrahpaşa, İstanbul Üniversitesi, Cerrahpaşa, Estambul, Turquía.

b. División de Nefrología Pediátrica, Departamento de Pediatría, Facultad de Medicina de İstanbul Üniversitesi, Cerrahpaşa, Estambul, Turquía.

c. División de Nefrología Pediátrica, Departamento de Pediatría, Hospital de Koç Üniversitesi, Estambul, Turquía.

d. División de Nefrología Pediátrica, Departamento de Pediatría, Facultad de Medicina de Koç Üniversitesi, Estambul, Turquía.

Correspondencia: Prof. Dr. Bülent Önal: bulonal@yahoo.com

Financiamiento:

Ninguno.

Conflicto de intereses: Ninguno que declarar.

Recibido: 16-5-2020

Aceptado: 10-9-2020

\section{RESUMEN}

Introducción. Los pediatras, cirujanos y subespecialistas, como urólogos y nefrólogos pediátricos, participan en el diagnóstico y tratamiento de la nefrolitiasis pediátrica. El objetivo fue determinar los enfoques de distintas disciplinas y evaluar las diferencias en sus protocolos de diagnóstico y tratamiento habituales.

Población y métodos. Cuestionario administrado a participantes de sesiones sobre nefrolitiasis en congresos nacionales en 2017 para evaluar las rutinas de diagnóstico y tratamiento de la nefrolitiasis entre distintas especialidades (cirujanos y pediatras) y subespecialidades (nefrólogos pediátricos y urólogos pediátricos). Resultados. Se analizaron 324 cuestionarios de 88 pediatras, 121 urólogos, 23 cirujanos pediátricos, 54 nefrólogos pediátricos $\mathrm{y}$ 38 urólogos pediátricos. Ambos grupos coincidieron en la necesidad de una evaluación metabólica. Para los cálculos ureterales distales $\geq 6 \mathrm{~mm}$, los cirujanos preferían una ureteroscopía; los pediatras, una litotricia por ondas de choque (LOC) $(p<0,001)$; y los subespecialistas, una ureteroscopía $(p=0,636)$. Para los cálculos en la parte inferior de los cálices renales $<1 \mathrm{~cm}$, los cirujanos y los subespecialistas preferían la LOC y los pediatras, la hidratación $(p<0,001$; $p=0,371$ ). Para los cálculos de entre $1,1 \mathrm{~cm}$ y $2 \mathrm{~cm}$, los cirujanos preferían la cirugía retrógrada intrarrenal (CRIR) y la LOC, y los pediatras, la LOC $(p=0,001)$. Para los cálculos más grandes, los cirujanos y subespecialistas preferían la nefrolitotomía percutánea (NLP) y los pediatras, la LOC ( $p=0,458 ; p=0,001)$.

Conclusión. Existen diferencias entre las disciplinas que participan activamente en el diagnóstico y tratamiento de la nefrolitiasis.

Palabras clave: urolitiasis, niño, ureteroscopía, educación, tratamientos.

http:/ / dx.doi.org/10.5546/ aap.2021.83

Texto completo en inglés:

http: / / dx.doi.org/10.5546/aap.2021.eng.83

Cómo citar: Önal B, Kirli EA, Canpolat N, Taşdemir M, et al. Distintos enfoques adoptados por los médicos para tratar la nefrolitiasis en pediatría: estudio basado en una encuesta. Arch Argent Pediatr 2021;119(2):83-90.

\section{INTRODUCCIÓN}

Si bien la nefrolitiasis pediátrica en general se considera una enfermedad relativamente rara, es endémica en algunas partes del mundo. ${ }^{1}$ Los pediatras, cirujanos y otros subespecialistas, como urólogos y nefrólogos pediátricos, participan en el diagnóstico y tratamiento de la nefrolitiasis. ${ }^{1}$ Según nuestra experiencia clínica, si bien todas las disciplinas que tienen una función activa en el tratamiento de la nefrolitiasis siguen los mismos lineamientos, nomogramas y manuales, en la práctica diaria, se observan diferencias. Aún no se han evaluado tales diferencias en los procedimientos diagnósticos y los enfoques de tratamiento según las distintas disciplinas. Sin embargo, las diferencias sustanciales en el conocimiento y la práctica pueden afectar, a su vez, la tasa de requisitos de intervención y resultados.

El objetivo de este estudio era determinar los enfoques de diagnóstico y tratamiento de las distintas disciplinas que tratan la nefrolitiasis y evaluar si existen diferencias en sus protocolos de diagnóstico y tratamiento habituales.

\section{POBLACIÓN Y MÉTODOS}

Una vez obtenida la aprobación institucional de este estudio (núm. 1456-822), se elaboró un cuestionario de 13 preguntas destinado a los médicos de distintas disciplinas que participan activamente en el 
diagnóstico, el tratamiento y el seguimiento de la nefrolitiasis pediátrica.

Antes de elaborar las preguntas de la encuesta, se revisó la bibliografía actual referida a la nefrolitiasis pediátrica y se determinaron las principales diferencias significativas. Dos urólogos (OÖ, AG), dos urólogos pediátricos (BÖ, EAK) y dos nefrólogos pediátricos (SÇ, NC) elaboraron las preguntas y los escenarios. Antes del estudio, un grupo de especialistas (pediatras y cirujanos) y subespecialistas (nefrólogos pediátricos y urólogos pediátricos) con distintos años de experiencia respondieron las preguntas en una división de nefrolitiasis de una sesión conjunta (2017, Estambul, Turquía). Luego los autores revisaron si quienes respondieron el cuestionario habían comprendido y completado las preguntas.

El cuestionario se administró a los participantes de las divisiones de nefrolitiasis pediátrica del Congreso Nacional de Urología (2017, Antalya, Turquía), el Congreso Nacional de Pediatría (2017, Estambul, Turquía) y el Congreso Nacional de Cirugía Pediátrica (2017, Antalya, Turquía).

Los participantes se agruparon en pediatras y cirujanos (urólogos, cirujanos pediátricos) para determinar la diferencia entre las especialidades. Los urólogos y cirujanos pediátricos que habían recibido formación oficial en urología pediátrica se consideraron urólogos pediátricos. De manera similar, los pediatras que habían recibido instrucción formal en nefrología pediátrica se consideraron nefrólogos pediátricos. Los urólogos pediátricos y los nefrólogos pediátricos tienen programas educativos comunes en cuanto a la nefrolitiasis (reuniones conjuntas de rutina, sesiones bibliográficas y clases magistrales) durante el internado posterior a la residencia.

Las primeras nueve preguntas buscaban determinar las características generales de los participantes en ambos grupos; las restantes empleaban escenarios típicos con los cuales los médicos podrían encontrarse en sus prácticas diarias para evaluar los métodos diagnósticos, terapéuticos y de seguimiento.

Los escenarios correspondían a tres situaciones diferentes relacionadas con la nefrolitiasis. El primer escenario consultaba sobre el enfoque adoptado para la microlitiasis renal (MR) (manchas de $<3 \mathrm{~mm}$ de diámetro detectadas en ecografía renal se han descripto como MR).

TABla 1. Características demográficas de los participantes

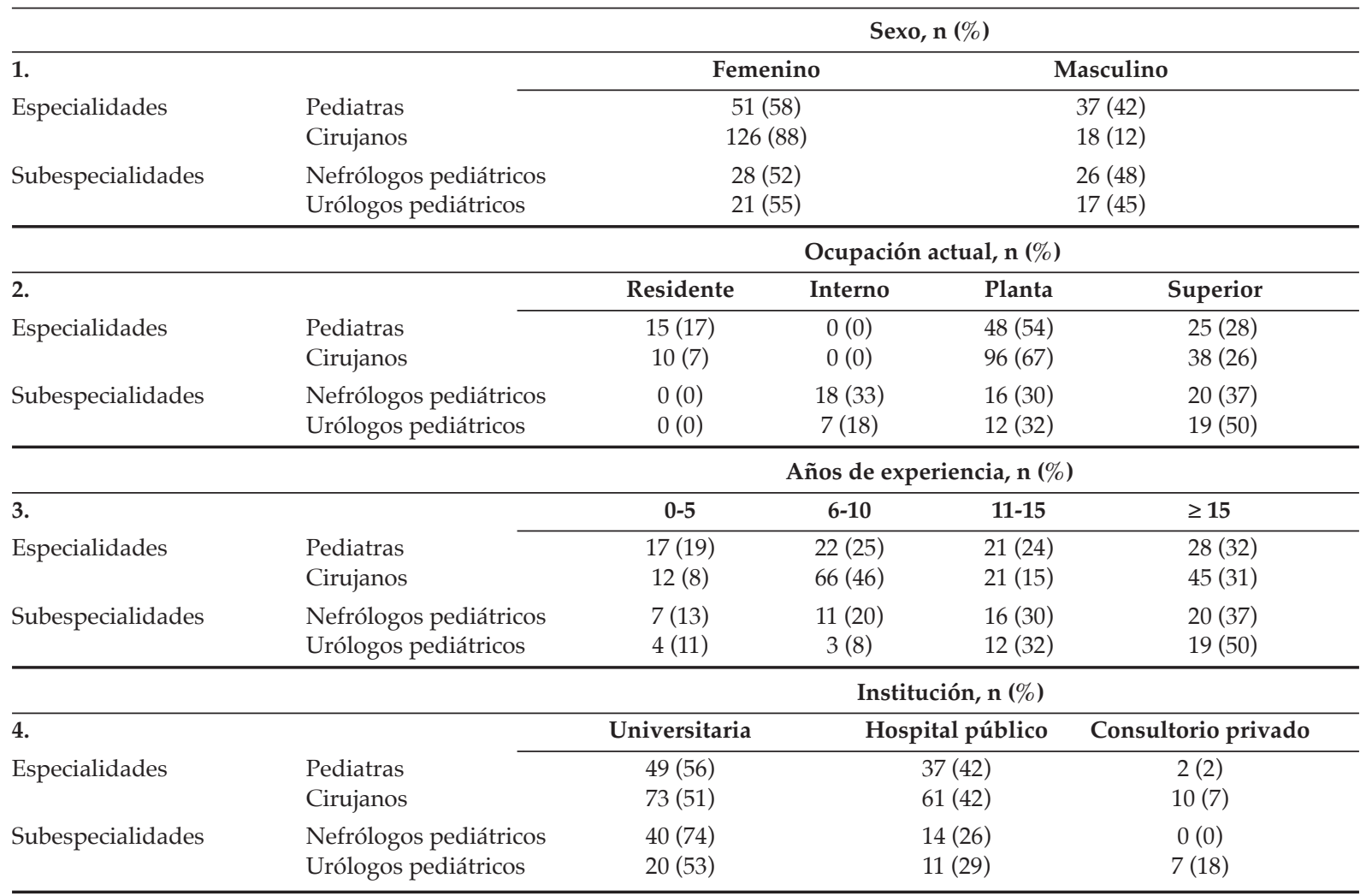


Los otros dos escenarios se establecieron según distintos tamaños de cálculos ubicados en la parte inferior de los cálices renales y en la parte distal de los uréteres.

Se incluyeron los cuestionarios completados con una única respuesta por pregunta y se excluyeron aquellos con varias respuestas por pregunta.

Los análisis estadísticos se hicieron con el programa SPSS, versión 13.0 (IBM Corp., Armonk, NY, EE. UU.). Se usaron la prueba $\chi^{2} y$ la prueba de Kruskal-Wallis (para la comparación de múltiples grupos) para la evaluación estadística. Un valor de $p<0,05$ se definió como estadísticamente significativo.

\section{RESULTADOS}

En total, de los 348 médicos que participaron en la encuesta, se analizaron 324 cuestionarios (después de la exclusión). Los especialistas fueron 88 pediatras (27\%), 121 urólogos $(37 \%)$ y 23 cirujanos pediátricos $(7 \%)$. Los subespecialistas fueron 54 nefrólogos pediátricos (17\%) y 38 urólogos pediátricos (12\%) (Figura 1). Las especialidades se dividieron en dos grupos: pediatras $(\mathrm{n}=88)$ y cirujanos $(\mathrm{n}=144)$; sus respuestas se evaluaron según su especialización. También se compararon las respuestas por subespecialidad. En la Tabla 1, se resumen las características demográficas de los participantes.

\section{Preguntas sobre la evaluación metabólica}

Si bien la evaluación diagnóstica de los participantes varió en la práctica diaria, los especialistas y los subespecialistas estuvieron de acuerdo en la necesidad de una evaluación metabólica. Si bien los pediatras hacían más evaluaciones metabólicas que los cirujanos (el $83 \%$ frente al $53 \%$ ), esta tasa fue similar entre los subespecialistas (el $98 \%$ frente al $95 \%$ ). Una mayor cantidad de cirujanos preferían el análisis de orina de 24 horas antes que los análisis de orina con dos muestras puntuales, en comparación con los pediatras (el $44 \%$ frente al $66 \%$, p: 0,032). Los subespecialistas coincidieron en realizar el análisis de orina de 24 horas (el $72 \%$ frente al $76 \%, p$ : 0,299). Los participantes que no hacían una evaluación metabólica indicaron mayormente como motivo la falta de capacitación. Además, los pediatras recomendaban que los niños con nefrolitiasis debían recibir seguimiento por parte de nefrólogos pediátricos (el $52 \%$ frente al $19 \%, p: 0,001)$, mientras que los subespecialistas coincidieron en que el seguimiento debía estar a cargo de un equipo multidisciplinario (el $73 \%$ frente al $74 \%, p: 0,657$ ) (Tabla 2).

\section{Preguntas sobre los escenarios clínicos}

Se preguntó a los participantes cómo abordaban la MR infantil. La mayoría de los especialistas preferían no hacer la prueba de nitroprusiato sódico en la evaluación de la MR (el $87 \%$ frente al $81 \%, p: 0,113$ ). Por el contrario, los subespecialistas estuvieron de acuerdo en hacer la prueba de nitroprusiato sódico en la evaluación de la MR (el $85 \%$ frente al $74 \%, p$ : $0,252)$. La evaluación radiológica de la MR no difirió entre los grupos, mientras que la ecografía fue la herramienta más preferida tanto por los especialistas (el $76 \%$ frente al $67 \%, p: 0,09)$ como

FIgURA 1. Diagrama de flujo de los participantes

Cantidad total de participantes invitados a completar la encuesta en los congresos nacionales $n=420$.

Urólogos $n=130$, pediatras $n=130$, cirujanos pediátricos $n=60$,

nefrólogos pediátricos $n=60$, urólogos pediátricos $n=40$.

Cantidad total de participantes que aceptaron participar $\mathrm{n}=348$.

Urólogos $n=129$, pediatras $n=92$, cirujanos pediátricos $n=35$, nefrólogos pediátricos $n=58$, urólogos pediátricos $n=40$.

Cantidad total de encuestas eliminadas $\mathrm{n}=24$.

Urólogos $n=8$, pediatras $n=4$, cirujanos pediátricos $n=12$, nefrólogos pediátricos $n=4$, urólogos pediátricos $n=2$.

Urólogos $n=121$, pediatras $n=88$, cirujanos pediátricos $n=23$, nefrólogos pediátricos $n=54$, urólogos pediátricos $n=38$. 
TABla 2. Enfoque de la elaboración del diagnóstico según las especialidades

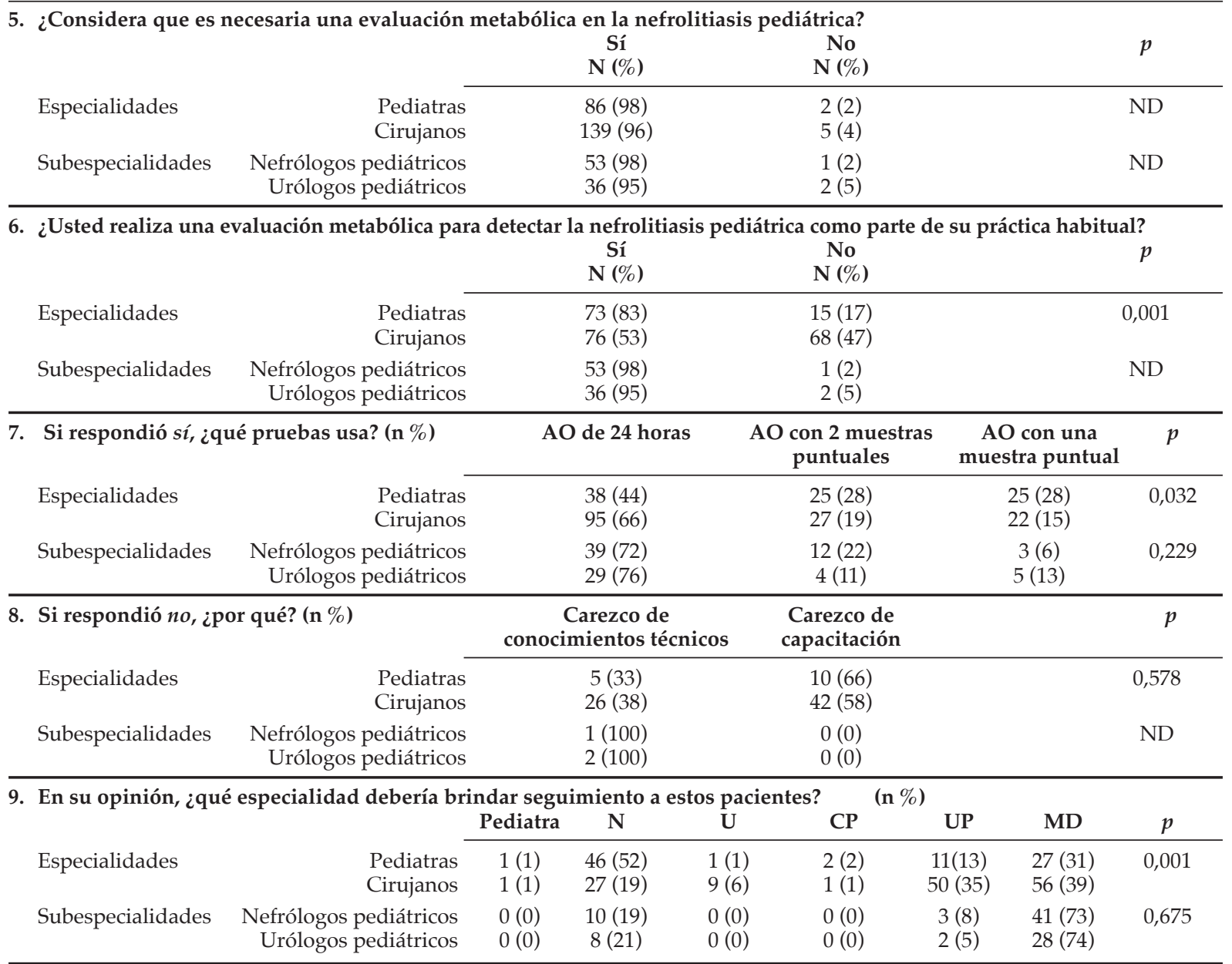

MD: multidisciplinario; N: nefrólogo; CP: cirujano pediátrico; UP: urólogo pediátrico; U: urólogo; AO: análisis de orina; ND: no disponible.

TABLA 3. Enfoque de la evaluación y la modalidad de tratamiento de la microlitiasis renal $(M R)$ según la especialidad 10. A un bebé de 3 meses de edad se le diagnosticó MR por ecografía.

\begin{tabular}{|c|c|c|c|c|c|c|c|c|c|}
\hline \multicolumn{4}{|c|}{ 10a ¿Evalúa al bebé mediante una prueba de nitroprusiato sódico en orina? } & \multicolumn{2}{|c|}{ Sí, n (\%) } & \multicolumn{3}{|c|}{ No, $\mathbf{n}(\%)$} & $p$ \\
\hline Especialidades & $\begin{array}{l}\text { Pediatras } \\
\text { Cirujanos }\end{array}$ & & & \multicolumn{2}{|c|}{$\begin{array}{l}11(13) \\
27(19)\end{array}$} & \multicolumn{3}{|c|}{$\begin{array}{l}77(87) \\
117(81)\end{array}$} & 0,113 \\
\hline Subespecialidades & $\begin{array}{l}\text { Nefrólogos pediátricos } \\
\text { Urólogos pediátricos }\end{array}$ & & & \multicolumn{2}{|c|}{$\begin{array}{l}46(85) \\
29(74)\end{array}$} & \multicolumn{3}{|c|}{$\begin{array}{l}8(15) \\
9(26)\end{array}$} & 0,252 \\
\hline \multicolumn{2}{|c|}{ 10b ¿Qué método radiológico prefiere a continuación? } & TC & PIV & \multicolumn{2}{|c|}{$\begin{array}{l}\text { UD } \\
\text { Ecografía }\end{array}$} & Ecografía & \multicolumn{2}{|c|}{ UD } & $p$ \\
\hline Especialidades & $\begin{array}{l}\text { Pediatras } \\
\text { Cirujanos }\end{array}$ & $\begin{array}{c}4(3) \\
13(7)\end{array}$ & $\begin{array}{l}1(1) \\
5(3)\end{array}$ & \multicolumn{2}{|c|}{$\begin{array}{l}26(18) \\
38(21)\end{array}$} & $\begin{array}{l}3(2) \\
4(2)\end{array}$ & \multicolumn{2}{|c|}{$\begin{array}{l}108(76) \\
122(67)\end{array}$} & 0,09 \\
\hline Subespecialidades & $\begin{array}{r}\text { Nefrólogos pediátricos } \\
\text { Urólogos pediátricos }\end{array}$ & $\begin{array}{l}0(0) \\
0(0)\end{array}$ & $\begin{array}{l}0(0) \\
0(0)\end{array}$ & \multicolumn{2}{|c|}{$\begin{array}{l}9(17) \\
8(21)\end{array}$} & $\begin{array}{l}0(0) \\
0(0)\end{array}$ & \multicolumn{2}{|c|}{$\begin{array}{l}45(83) \\
30(79)\end{array}$} & 0,672 \\
\hline \multicolumn{2}{|c|}{ 10c ¿Qué modalidad de tratamiento prefiere para este bebé? } & $\begin{array}{l}\text { Abordaje } \\
\text { quirúrgico }\end{array}$ & \multicolumn{2}{|c|}{$\begin{array}{c}\text { Ecografía } \\
\text { Seguimiento }\end{array}$} & & $\begin{array}{l}\text { ratación } \\
\text { imiento }\end{array}$ & Multi & Ignorar & $p$ \\
\hline Especialidades & $\begin{array}{l}\text { Pediatras } \\
\text { Cirujanos }\end{array}$ & $\begin{array}{l}1(1) \\
6(4)\end{array}$ & \multicolumn{2}{|c|}{$\begin{array}{l}42(48) \\
70(49)\end{array}$} & & $\begin{array}{l}36(41) \\
53(37)\end{array}$ & $\begin{array}{c}8(9) \\
14(10)\end{array}$ & $\begin{array}{l}1(1) \\
1(1)\end{array}$ & 0,956 \\
\hline Subespecialidades & $\begin{array}{l}\text { Nefrólogos pediátricos } \\
\text { Urólogos pediátricos }\end{array}$ & $\begin{array}{l}0(0) \\
0(0)\end{array}$ & \multicolumn{2}{|c|}{$\begin{array}{l}25(46) \\
17(45)\end{array}$} & & $\begin{array}{l}24(44) \\
19(50)\end{array}$ & $\begin{array}{l}5(9) \\
2(5)\end{array}$ & $\begin{array}{l}0(0) \\
0(0)\end{array}$ & 0,732 \\
\hline
\end{tabular}

TC: tomografía computada; UD: urografía directa; PIV: pielografía intravenosa. 
por los subespecialistas (el $83 \%$ frente al $79 \%$, $p: 0,672)$. Ambos grupos estuvieron de acuerdo en las modalidades de tratamiento, y las tasas de seguimiento mediante ecografía y con hidratación fueron casi idénticas (Tabla 3).

Asimismo, a los participantes se les consultó sobre su abordaje de los cálculos sintomáticos ureterales distales sin complicaciones. La mayoría de los participantes de ambos grupos elegían un procedimiento similar para los cálculos en la parte distal de los uréteres con un diámetro de $3 \mathrm{~mm}$ a $5 \mathrm{~mm}$. Entre los especialistas, las opciones de tratamiento de los cálculos en la parte distal de los uréteres que medían $\geq 6 \mathrm{~mm}$ variaban: mientras que los cirujanos (52\%) preferían la ureteroscopía; los pediatras $(36 \%)$, la litotricia por ondas de choque (LOC) $(p<0,001$; Tabla 4$)$. Sin embargo, los subespecialistas preferían la ureteroscopía como tratamiento (el $66 \%$ frente al $68 \%, p: 0,636)$.

Se preguntó a los participantes sobre el tratamiento de los cálculos de distintos tamaños en la parte inferior de los cálices renales. Si el cálculo medía $<1 \mathrm{~cm}$, la primera opción de los cirujanos era la LOC, mientras que los pediatras optaban por la hidratación (el $54 \%$ frente al $40 \%, p<0,001)$. Los subespecialistas preferían la LOC como tratamiento de primera línea (el $55 \%$ frente al $55 \%, p: 0,371)$. Si un cálculo medía entre $1,1 \mathrm{~cm}$ y $2 \mathrm{~cm}$, los cirujanos preferían la cirugía retrógrada intrarrenal (CRIR) $(33 \%)$ y la LOC (33 \%) casi por igual, pero la mayoría de los pediatras prefería la LOC $(40 \%)(p: 0,001)$. Si un cálculo medía $>2 \mathrm{~cm}$, la mayoría de los cirujanos prefería la nefrolitotomía percutánea (NLP) (78\%), mientras que los pediatras preferían la LOC (51\%) como primera opción y la NLP $(33 \%)$ como segunda opción ( $p: 0,001)$. Los subespecialistas preferían la NLP como tratamiento (el $56 \%$ frente al $63 \%, p: 0,458$ ).

También se observó esta diferencia en la selección de los métodos radiológicos de diagnóstico por imagen. Los participantes de ambas especialidades preferían la radiografía simple de riñones, uréteres y vejiga (RUV) más una ecografía en lugar de la ecografía sola. Sin embargo, la mitad de los urólogos pediátricos $(53 \%)$ optaban por la tomografía computada (TC) de baja dosis y los nefrólogos pediátricos preferían la urografía directa (UD) y la ecografía $(46 \%)$ como instrumento de diagnóstico $(p: 0,029)$ (Tabla 5).

\section{DISCUSIÓN}

El manejo de la nefrolitiasis pediátrica abarca múltiples disciplinas. Los estudios previos encontrados en la bibliografía se centraron principalmente en los enfoques diagnósticos y las

TABLA 4. Modalidades de diagnóstico y tratamiento según los distintos tamaños de los cálculos en la parte distal de los uréteres

\begin{tabular}{|c|c|c|c|c|c|c|c|}
\hline \multicolumn{8}{|c|}{ 11. Cálculo sintomático ureteral distal sin complicaciones en un niño de 5 años } \\
\hline \multicolumn{5}{|c|}{ 11a. ¿Qué modalidad de tratamiento prefiere si el cálculo mide $3 \mathrm{~mm}$ ? } & $\begin{array}{l}\text { Hidratación } \\
+ \text { TME }\end{array}$ & $\begin{array}{c}\text { n (\%) } \\
\text { Hidratación }\end{array}$ & $p$ \\
\hline \multirow[t]{2}{*}{ Especialidades } & Pediatras & $1(1)$ & $1(1)$ & $2(2)$ & $10(11)$ & $74(84)$ & 0,545 \\
\hline & Cirujanos & $2(1)$ & $5(3)$ & $3(2)$ & $50(35)$ & $83(59)$ & \\
\hline \multirow[t]{2}{*}{ Subespecialidades } & Nefrólogos pediátricos & $0(0)$ & $0(0)$ & $1(1)$ & $18(33)$ & $35(66)$ & 0,583 \\
\hline & Urólogos pediátricos & $0(0)$ & $0(0)$ & $0(0)$ & $14(37)$ & $24(63)$ & \\
\hline \multirow{2}{*}{\multicolumn{4}{|c|}{$\begin{array}{r}\text { 11b. ¿Qué modalidad de tratamiento prefiere si el cálculo mide } 5 \mathrm{~mm} \text { ? } \\
\text { Endoprótesis Ureteroscopía }\end{array}$}} & & Hidratación & n $(\%)$ & $p$ \\
\hline & & & & LOC & + TME & Hidratación & \\
\hline \multirow{2}{*}{ Especialidades } & Pediatras & $4(5)$ & $3(3)$ & $21(24)$ & $46(52)$ & $14(16)$ & 0,112 \\
\hline & Cirujanos & $1(1)$ & $28(19)$ & $17(12)$ & $60(42)$ & $38(26)$ & \\
\hline \multirow[t]{2}{*}{ Subespecialidades } & Nefrólogos pediátricos & $2(4)$ & $11(20)$ & $3(6)$ & $25(46)$ & $13(24)$ & 0,641 \\
\hline & Urólogos pediátricos & $0(0)$ & $14(37)$ & $1(3)$ & $13(34)$ & $10(26)$ & \\
\hline \multirow{2}{*}{\multicolumn{4}{|c|}{$\begin{array}{l}\text { 11c. ¿Qué modalidad de tratamiento prefiere si el cálculo mide } \geq 6 \mathrm{~mm} \text { ? } \\
\text { Endoprótesis Ureteroscopía }\end{array}$}} & & Hidratación & n $(\%)$ & $p$ \\
\hline & & & & LOC & + TME & Hidratación & \\
\hline \multirow[t]{2}{*}{ Especialidades } & Pediatras & $19(22)$ & $15(17)$ & $32(36)$ & $20(23)$ & $2(2)$ & $<0,001$ \\
\hline & Cirujanos & $11(8)$ & $75(52)$ & $28(19)$ & $17(12)$ & $13(9)$ & \\
\hline \multirow[t]{2}{*}{ Subespecialidades } & Nefrólogos pediátricos & $5(13)$ & $36(66)$ & $6(11)$ & $6(11)$ & $2(6)$ & 0,636 \\
\hline & Urólogos pediátricos & $6(16)$ & $26(68)$ & $1(3)$ & $3(8)$ & $2(5)$ & \\
\hline
\end{tabular}

TME: terapia médica de expulsión; LOC: litotricia por ondas de choque. 
opciones de tratamiento médico y quirúrgico que mejoran los resultados. No obstante, este estudio se centró en las diferencias en los enfoques de diagnóstico y tratamiento adoptados por las distintas disciplinas. Cabe destacar el resultado del estudio, que demuestra que los pediatras y cirujanos parecen tener enfoques diagnósticos y terapéuticos diferentes. Otra implicancia notable de este estudio es que los nefrólogos y los urólogos pediátricos adoptan enfoques diagnósticos y terapéuticos similares asociados con la nefrolitiasis.

La mayoría de los participantes de este estudio tenían más de cinco años de experiencia, lo que implica que conocen los lineamientos existentes sobre el manejo de la nefrolitiasis y, por lo tanto, están capacitados para tratar la urolitiasis complicada.

Según los lineamientos actuales, debido a la elevada incidencia de los factores predisponentes y a las altas tasas de recurrencia de litiasis, cada niño con urolitiasis debe someterse a una evaluación metabólica completa. ${ }^{1,3,6}$ A pesar de las diferencias en la práctica diaria, todos los participantes coincidieron en la importancia de la evaluación metabólica. Si bien los pediatras la hacen con mayor frecuencia que los cirujanos, prefieren el análisis de muestras puntuales de orina. Los subespecialistas y los cirujanos prefirieron el análisis de orina de 24 horas. El análisis de muestras puntuales de orina es sencillo en los niños, pero los resultados pueden ser engañosos. Esta orientación permitirá una mayor complejidad diagnóstica. Un punto importante mencionado por los especialistas es la falta de capacitación durante su formación, como motivo para omitir la evaluación metabólica; sin embargo, entre los subespecialistas, la falta de conocimientos técnicos suficientes fue el único motivo para no hacer las pruebas metabólicas. Asimismo, la disponibilidad de métodos diagnósticos y tratamientos podría variar entre el sector público y el privado y, en consecuencia, afectar el proceso de toma de

TABla 5. Modalidades de diagnóstico y tratamiento según los distintos tamaños de los cálculos en los cálices renales

12. Cálculo asintomático en la parte inferior de los cálices renales sin complicaciones en un niño de 5 años.

\begin{tabular}{|c|c|c|c|c|c|c|c|}
\hline 12a. ¿Qué modalidad d & ratamiento prefiere si el & $\begin{array}{l}\text { ulo mide } \\
\text { NLP }\end{array}$ & $\begin{array}{c}\text { nos de } 1 \\
\text { CRIR }\end{array}$ & LOC & $\begin{array}{l}\text { Hidratación } \\
\text { + TME }\end{array}$ & $\begin{array}{c}\text { n }(\%) \\
\text { Hidratación }\end{array}$ & $p$ \\
\hline Especialidades & $\begin{array}{l}\text { Pediatras } \\
\text { Cirujanos }\end{array}$ & $\begin{array}{l}2(2) \\
1(1)\end{array}$ & $\begin{array}{c}3(3) \\
14(10)\end{array}$ & $\begin{array}{l}13(15) \\
57(40)\end{array}$ & $\begin{array}{l}23(26) \\
26(18)\end{array}$ & $\begin{array}{l}47(54) \\
46(32)\end{array}$ & 0,001 \\
\hline Subespecialidades & $\begin{array}{l}\text { Nefrólogos pediátricos } \\
\text { Urólogos pediátricos }\end{array}$ & $\begin{array}{l}2(4) \\
3(8)\end{array}$ & $\begin{array}{c}1(2) \\
5(13)\end{array}$ & $\begin{array}{l}30(55) \\
21(55)\end{array}$ & $\begin{array}{c}11(20) \\
4(11)\end{array}$ & $\begin{array}{c}10(19) \\
5(13)\end{array}$ & 0,371 \\
\hline \multicolumn{5}{|c|}{ 12b. ¿Qué modalidad de tratamiento prefiere si el cálculo mide entre $1,1 \mathrm{~cm}$ y $2 \mathrm{~cm}$ ? } & $\begin{array}{l}\text { Hidratación } \\
\text { + TME }\end{array}$ & $\begin{array}{c}\text { n }(\%) \\
\text { Hidratación }\end{array}$ & $p$ \\
\hline Especialidades & $\begin{array}{l}\text { Pediatras } \\
\text { Cirujanos }\end{array}$ & $\begin{array}{c}7(8) \\
36(25)\end{array}$ & $\begin{array}{l}9(10) \\
48(33)\end{array}$ & $\begin{array}{l}35(40) \\
44(31)\end{array}$ & $\begin{array}{c}32(36) \\
12(8)\end{array}$ & $\begin{array}{l}5(6) \\
4(3)\end{array}$ & 0,001 \\
\hline Subes & $\begin{array}{r}\text { Nefrólogos pediátricos } \\
\text { Urólogos pediátricos }\end{array}$ & $\begin{array}{l}7(13) \\
6(16)\end{array}$ & $\begin{array}{l}7(13) \\
7(18)\end{array}$ & $\begin{array}{l}33(61) \\
23(60)\end{array}$ & $\begin{array}{l}5(9) \\
1(3)\end{array}$ & $\begin{array}{l}2(4) \\
1(3)\end{array}$ & 0,246 \\
\hline \multicolumn{5}{|c|}{ 12c. ¿Qué modalidad de tratamiento prefiere si el cálculo mide más de $2 \mathrm{~cm}$ ? } & $\begin{array}{l}\text { Hidratación } \\
\text { + TME }\end{array}$ & $\begin{array}{c}\text { n }(\%) \\
\text { Hidratación }\end{array}$ & $p$ \\
\hline Especialidades & $\begin{array}{l}\text { Pediatras } \\
\text { Cirujanos }\end{array}$ & $\begin{array}{c}29(33) \\
112(78)\end{array}$ & $\begin{array}{c}8(9) \\
17(12)\end{array}$ & $\begin{array}{c}45(51) \\
14(9)\end{array}$ & $\begin{array}{l}4(5) \\
2(1)\end{array}$ & $\begin{array}{l}2(2) \\
0(0)\end{array}$ & 0,001 \\
\hline Subespecialidades & $\begin{array}{r}\text { Nefrólogos pediátricos } \\
\text { Urólogos pediátricos }\end{array}$ & $\begin{array}{l}30(56) \\
24(63)\end{array}$ & $\begin{array}{l}12(19) \\
10(26)\end{array}$ & $\begin{array}{c}10(22) \\
4(11)\end{array}$ & $\begin{array}{l}2(2) \\
0(0)\end{array}$ & $\begin{array}{l}0(0) \\
0(0)\end{array}$ & 0,458 \\
\hline
\end{tabular}

13. Niño de 10 años ingresado a la sala de emergencias con síntomas de cólico renal. ¿Qué método de diagnóstico por imagen prefiere?

\begin{tabular}{lcccccc} 
& & \multicolumn{1}{c}{$\mathbf{n}(\mathbf{\%})$} & & \\
& & TC & UD Ecografía & UD & Ecografía & $p$ \\
\cline { 3 - 7 } Especialidades & Pediatras & $4(5)$ & $42(48)$ & $3(3)$ & $39(44)$ & 0,057 \\
\multirow{2}{*}{ Subespecialidades } & Cirujanos & $26(18)$ & $68(47)$ & $1(1)$ & $49(34)$ & \\
& Nefrólogos pediátricos & $5(9)$ & $25(46)$ & $3(6)$ & $21(39)$ & 0,029 \\
& Urólogos pediátricos & $20(53)$ & $9(24)$ & $2(5)$ & $7(18)$ & \\
\hline
\end{tabular}

TC: tomografía computada; UD: urografía directa; TME: terapia médica de expulsión; NLP: nefrolitotomía percutánea; CRIR: cirugía retrógrada intrarrenal. 
decisiones al seleccionar uno u otro método. Todos los motivos son factores de riesgo de un retraso en el diagnóstico de un trastorno metabólico subyacente.

Se han presentado pocos informes sobre el diagnóstico, la evaluación, el tratamiento, el seguimiento a largo plazo y los resultados de la MR en la bibliografía. Debido a la falta de estudios controlados, es poco clara la información sobre la incidencia real y los pasos que seguir en el diagnóstico y el tratamiento. ${ }^{8,9}$ En nuestro estudio, quedó demostrada esta falta de claridad, ya que ambos grupos prefirieron distintas evaluaciones diagnósticas y tratamientos conservadores para la MR, como la hidratación y el seguimiento estrecho mediante ecografía. La mayoría de los pacientes pediátricos con MR tienen uno o más trastornos metabólicos subyacentes que son similares a aquellos con nefrolitiasis. ${ }^{8,9}$ Si bien los subespecialistas estuvieron de acuerdo en que la prueba de nitroprusiato sódico es útil para la evaluación metabólica de primera línea, los especialistas coincidieron en que la evaluación metabólica no es un requisito. Este resultado podría ser alarmante porque este tema no ha sido lo suficientemente aclarado en la bibliografía y requiere que los médicos tomen precauciones.

La terapia médica de expulsión (TME), mayormente con bloqueantes $\alpha$, se recomendó para los cálculos sin complicaciones que medían $<10 \mathrm{~mm}$ ubicados en la parte distal de los uréteres. ${ }^{5} \mathrm{~A}$ modo de reflexión, los participantes coincidieron en la TME y la hidratación como modalidades de tratamiento de los cálculos pequeños en la parte distal de los uréteres, aunque en la bibliografía existen conflictos sobre el uso de la TME para una indicación no autorizada. ${ }^{10}$ Los especialistas también difirieron en el enfoque del tratamiento de los cálculos en la parte distal de los uréteres que miden $\geq 6 \mathrm{~mm}$. La LOC se ha utilizado para tratar los cálculos ureterales con tasas de éxito inferiores para los cálculos ureterales distales, y los pediatras tienden a insistir en el uso de la LOC o el manejo conservador; sin embargo, los cirujanos y ambos grupos de subespecialistas prefieren la ureteroscopía. Esta orientación se correlaciona con los lineamientos clínicos. ${ }^{1,11,12}$

Si bien la LOC se recomienda como primera línea de tratamiento para los cálculos $<10 \mathrm{~mm}$ en los cálices renales, ${ }^{1}$ los pediatras prefieren el tratamiento con hidratación, mientras que los cirujanos y los subespecialistas, la LOC. Este enfoque explica por qué la LOC no se aceptó completamente como tratamiento no invasivo entre los pediatras debido a la necesidad de sedoanalgesia o anestesia general en los niños. Recientemente, la CRIR se ha popularizado más debido a la mayor tasa de ausencia de litiasis en comparación con la LOC. ${ }^{1,13}$ Los cirujanos prefirieron la CRIR (33 \%) y la LOC (31\%) casi por igual para el tratamiento de los cálculos que miden entre $1,1 \mathrm{~cm}$ y $2 \mathrm{~cm}$. La mayoría de los cirujanos y subespecialistas prefirieron la NLP, pero los pediatras prefirieron igualmente, la LOC como primera opción para los cálculos de más de $2 \mathrm{~cm}$. Esta observación puede explicarse por el hecho de que los pediatras tienden a actuar en forma más conservadora que los cirujanos. Otra posible explicación es que la mayoría de los pediatras no están lo suficientemente familiarizados con estos procedimientos mínimamente invasivos.

Los especialistas y los nefrólogos pediátricos prefirieron la ecografía o la radiografía de RUV por igual en los niños con cólicos renales. Este resultado podría ser secundario al seguimiento de un protocolo de radiación -tan baja como sea razonable- (ALARA, sigla correspondiente en inglés) en los niños. ${ }^{14}$ Recientemente, en algunos estudios se informó que una TC de baja dosis es mejor que una ecografía para detectar los cálculos y clasificar la anatomía renal. ${ }^{15}$ Por lo tanto, la mitad de los urólogos pediátricos prefieren usar la TC de baja dosis para el diagnóstico. Esta preferencia entre los cirujanos podría deberse a que desean observar la anatomía renal y la carga y ubicación de la litiasis para facilitar el procedimiento quirúrgico.

Si bien los pediatras recomiendan que los nefrólogos pediátricos deben realizar el seguimiento de los niños con nefrolitiasis, los subespecialistas coinciden en un seguimiento con un enfoque multidisciplinario con más disciplinas especializadas, como urología y nefrología pediátricas.

A modo de resumen, los departamentos que se ocupan del tratamiento de la nefrolitiasis podrían tener distintos enfoques en relación con el diagnóstico y los protocolos de tratamiento. Esta diferencia podría relacionarse con la falta de evidencia o de lineamientos adecuados y la rápida evolución de la tecnología. Además, el conflicto de intereses inherente que un especialista podría tener para preferir cierta modalidad diagnóstica y terapéutica podría ser otro motivo de las distintas elecciones. También podría estar relacionada con la carga asistencial. Una mayor cantidad 
de pacientes con litiasis llevan a una mayor experiencia y familiaridad con la bibliografía actual. En esta encuesta, los subespecialistas dieron respuestas similares a los escenarios clínicos y las preguntas sobre la evaluación metabólica. Tal similitud podría ser resultado de los programas de formación comunes, que ofrecen un manejo integral de la nefrolitiasis pediátrica por parte de estas disciplinas y les permiten trabajar como un equipo especializado en litiasis.

Una de las limitaciones de este estudio es que los datos se obtuvieron de un cuestionario de respuestas múltiples y los participantes se vieron obligados a adaptar sus conceptos a las opciones preparadas en forma simplificada. Por lo tanto, los resultados tal vez no reflejen la práctica diaria con precisión. Otra limitación es que el cuestionario no estaba validado, lo que podría generar un sesgo potencial entre los encuestados. Además, los hallazgos corresponden solamente a los encuestados a disposición de los autores. Estos podrían ser representativos de la población del estudio, pero tal vez no sea adecuado generalizar los resultados del estudio a otras poblaciones. A pesar de estas limitaciones, nuestros resultados revelan las diferencias en los enfoques de diagnóstico, tratamiento y seguimiento de los médicos de distintas disciplinas que podrían tener una participación activa en la nefrolitiasis pediátrica. La preferencia de distintas modalidades de diagnóstico y tratamiento entre las disciplinas podría afectar los procedimientos diagnósticos y las modalidades de tratamiento y llevar a una menor tasa de ausencia de litiasis y una mayor tasa de recurrencia.

\section{CONCLUSIÓN}

Existen diferencias entre las disciplinas que participan activamente en el diagnóstico y tratamiento de la nefrolitiasis.

La integración de las disciplinas en el tratamiento de la nefrolitiasis pediátrica y el establecimiento de equipos dedicados a las litiasis podrían ayudar a reducir los conflictos en los enfoques de diagnóstico y tratamiento.

\section{REFERENCIAS}

1. Tekgül S, Doğan HS, Hoebeke P, Kocvara R, et al. Urinary stone disease. En: EAU Guidelines on Pediatric Urology. 2016;58-65. [Fecha de acceso: 10 de septiembre de 2020]. Disponibleen:https: / / uroweb.org/wp-content/uploads / EAU-Guidelines-Paediatric-Urology-2016.pdf.

2. Assimos D, Krambeck A, Miller N.L, Monga M, et al. Surgical management of stones: American Urological Association/Endourological Society guideline. Part I. J Urol. 2016; 196(4):1153-60.

3. Radmayr C, BogaertG, Doğan HS, Nijman JM, et al. Urinary Stone disease. En: EAU Guidelines on Pediatric Urology. 2020: 65-74. [Fecha de acceso: 10 de septiembre de 2020]. Disponibleen:https: / / uroweb.org/guideline/ paediatricurology /

4. SchneckFX, OstM. Surgical management of pediatric stone disease. En: Wein AJ, Kavoussi LR, Partin AW, Peter CA. Cambell-Walsh Urology. $11^{\text {th }}$ ed. Philadelphia: Elsevier; 2016.p.3102-20.

5. Türk C, Petrik A, Sarica K, Seitz C et al. EAU guidelines on diagnosis and conservative management of urolithiasis. Eur Urol. 2016; 69(3):468-74.

6. Straub, M. Strohmaier WL, Berg W, Beck B et al. Diagnosis and metaphylaxis of stone disease. Consensus concept of the National Working Committee on Stone Disease for the upcoming German Urolithiasis Guideline. World J Urol. 2005; 23(5):309-23.

7. Liu W, Esler SJ, Kenny BJ, Goh RH, et al. Low-dose nonenhanced helical CT of renal colic: assessment of ureteric stone detection and measurement of effective dose equivalent. Radiology. 2015; (1):51-4.

8. Fallahzade MA, Hassanzadeh J, Fallahzadeh MH. What do we know about pediatric renal microlithiasis? J Renal Inj Prev. 2017; (6):70-5.

9. Bilge I, Yılmaz A, Kayıran SM, Emre S, et al. Clinical importance of renal calyceal microlithiasis in children. Pediatr Int. 2013; 55(6):731-6.

10. Knoll T, Türk C. The role of medical expulsive therapy for ureteral stones: pro MET. Eur Urol Focus. 2017; 3(1):3-4.

11. Onal, B, Demirkesen O, Tansu N, Kalkan M, et al. The impact of caliceal pelvic anatomy on stone clearance after shock wave lithotripsy for pediatric lower pole stones. J Urol. 2004; 172(3):1082-6.

12. Hochreiter WW, Danuser H, Perrig M, Studer UE. Extracorporeal shock wave lithotripsy for distal ureteral calculi: what a powerful machine can achieve. J Urol. 2003; 169(3):878-80.

13. Ateş F, Zor M, Yılmaz O, Tuncer M, et al. Management behaviors of the urology practitioners to the small lower calyceal stones: the result of a web-based survey. Urolithiasis. 2016; 44(3):277-81.

14. Sodhi KS, Krishna S, Saxena AK, Sinha A, et al. Clinical application of 'justification' and 'optimization' principle of ALARA in pediatric CT imaging: "How many children can be protected from unnecessary radiation?" Eur J Radiol. 2015; 84(9):1752-7.

15. Passerotti C, Chow JS, Silva A, Schoettler CL, et al. Ultrasound versus computerized tomography for evaluating urolithiasis. J Urol. 2009; 182(4 Suppl):1829-34. 